\title{
Templated Synthesis and Assembly of Two-, Three- and Six-Patch Silica Nanoparticles with a Controlled Patch-to-Particle Size Ratio
}

\author{
Bin Liu ${ }^{1,2}\left(\mathbb{D}\right.$, Stéphanie Exiga ${ }^{1}$, Etienne Duguet ${ }^{2, *(\mathbb{D})}$ and Serge Ravaine ${ }^{1, *(\mathbb{D})}$ \\ 1 Univ. Bordeaux, CNRS, CRPP, UMR 5031, 33600 Pessac, France; bin.liu-bin@u-bordeaux.fr (B.L.); \\ stephanie.exiga@crpp.cnrs.fr (S.E.) \\ 2 Univ. Bordeaux, CNRS, Bordeaux INP, ICMCB, UMR 5026, 33600 Pessac, France \\ * Correspondence: etienne.duguet@icmcb.cnrs.fr (E.D.); serge.ravaine@crpp.cnrs.fr (S.R.)
}

Citation: Liu, B.; Exiga, S.; Duguet, E.; Ravaine, S. Templated Synthesis and Assembly of Two-, Three- and Six-Patch Silica Nanoparticles with a Controlled Patch-to-Particle Size Ratio. Molecules 2021, 26, 4736. https://doi.org/10.3390/ molecules26164736

Academic Editor: Nadine Millot

Received: 3 July 2021

Accepted: 3 August 2021

Published: 5 August 2021

Publisher's Note: MDPI stays neutral with regard to jurisdictional claims in published maps and institutional affiliations.

Copyright: (c) 2021 by the authors. Licensee MDPI, Basel, Switzerland. This article is an open access article distributed under the terms and conditions of the Creative Commons Attribution (CC BY) license (https:// creativecommons.org/licenses/by/ $4.0 /)$.

\begin{abstract}
We report a fabrication route of silica nanoparticles with two, three or six patches with an easily tunable patch-to-particle size ratio. The synthetic pathway includes two main stages: the synthesis of silica/polystyrene multipod-like templates and the selective growth of their silica core through an iterative approach. Electron microscopy of the dimpled nanoparticles obtained after dissolution of the polystyrene nodules of the multipod-like nanoparticles provides evidence of the conformational growth of the silica core. Thanks to the presence of some polymer chains, which remained grafted at the bottom of the dimples after the dissolution of the PS nodules, the solventinduced assembly of the patchy nanoparticles is performed. Chains, hexagonal suprastructures and cubic lattices are obtained from the assembly of two-, three- and six-patch silica nanoparticles, respectively. Our study can guide future work in both patchy nanoparticle synthesis and selfassembly. It also opens new routes towards the fabrication of specific classes of one-, two- and three-dimensional colloidal lattices, including complex tilings.
\end{abstract}

Keywords: patchy nanoparticles; silica; patch size; assembly

\section{Introduction}

In the last decades, tremendous efforts have been made towards the development of synthetic strategies for hybrid organic-inorganic (nano)materials, on the one hand [1-3], and for anisotropic particles with well-controlled size, shape and composition, on the other hand [4-11]. Most of the latter has been aimed at producing building blocks for the bottom-up construction of novel functional materials and devices with more complex and predictable architectures, which could have applications in photonics [12] and optics [13]. One of the most effective strategies towards achieving high degrees of synthetic control consists of using a pre-existing template. For instance, emulsion droplets have been often used as template to synthesize hollow particles: silica capsules have been prepared from Pickering emulsions [14] or in oil-in-water emulsions [15], and iron oxide hollow spheres have been synthesized in glycerol-in-water quasi-emulsion [16]. Colloidal particles can also serve as a template to synthesize hollow or hybrid structures. As an example, Xia et al. demonstrated the conformational coating of positively charged polystyrene (PS) beads with silica [17]. They also reported that when negatively charged PS beads were employed as templates, the silica coating was not homogeneous and silica colloids with non-spherical morphologies were produced. Titania hollow spheres were obtained through deposition of titania on the surface of silica particles by hydrolysis of titanium butoxide and condensation with the assistance of hydroxypropyl cellulose, which served as a surfactant [18]. Chen et al. demonstrated the formation of Janus particles by modifying the surface of gold nanoparticles with poly-acrylic acid and 4-mercaptophenylacetic acid acting as competing surfactants [19]. Janus particles can be considered as one-patch colloids if one of the two 
hemispheres is sticky. More generally, multi-patch particles are very interesting for their ability to assemble with their fellows by controlling the number of particles in contact and the orientation of the bonds according to the well-known valence principle for atoms in covalent compounds [20]. We reported that multivalent silica particles with well-controlled size and shape could be obtained from multipod-like silica/PS nanoparticles acting as templates through the slow addition of an ethanol solution of tetraethoxysilane (TEOS), which induced the conformational growth of their silica core [21]. More recently, we showed that the continuous addition of larger quantities of TEOS did not allow us to extend the re-growth of the silica core of tetrapod-like particles. Indeed, TEOS molecules migrate inside the PS nodules when their concentration is too high, leading to the formation of excrescences on the surface of the nodules [22]. To avoid the undesired formation of these silica bumps, we developed an alternative iterative route based on successive additions of a small amount of TEOS and centrifugation/redispersion cycles to eliminate the excess of unreacted TEOS.

Here, we report that we successfully extended this strategy to bi-, tri- and hexapod-like templates, allowing us to fabricate silica particles with 2, 3 or 6 PS patches, respectively. By carefully analyzing the particles by electron microscopy, we show that we could precisely control the patch-to-particle size ratio by varying the added amount of TEOS. The specific dissolution of PS gave rise to silica nanoparticles with a precise number of dimples, which combine both enthalpic and entropic patchiness thanks to the presence of grafted PS chains at the bottom of the cavities [23]. Taking advantage of the stickiness of the latter in a good/bad solvent mixture, we successfully assembled the patchy nanoparticles into chains, hexagonal suprastructures and simple cubic lattices.

\section{Results}

Scheme 1 shows the multi-step approach developed to synthesize patchy silica particles with controlled patch size from PS/silica multipods. It consists of growing the silica core of the silica/PS multipods used as templates through successive additions of a small amount of TEOS interspersed with centrifugation/redispersion cycles in order to eliminate the excess of unreacted TEOS molecules and the formation of silica protuberances at the surface of the PS lobes, which occurs when TEOS is added at once [22].

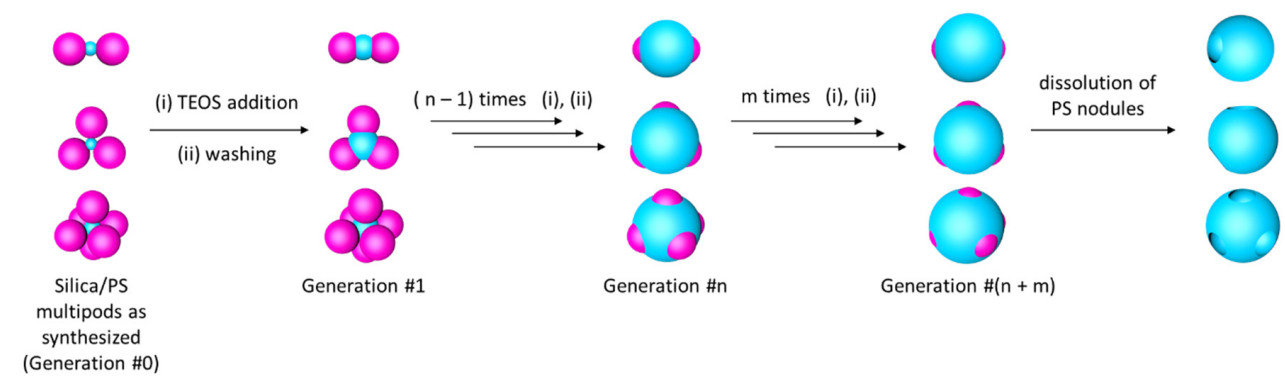

Scheme 1. Synthetic route for the preparation of particles with two-, three- and six-PS patches of controlled size from the corresponding silica/PS multipods and the subsequent formation of particles with concave patches through dissolution of the PS lobes.

Transmission electron microscopy (TEM) images in Figure 1a,g,m show the morphology of the bi-, tri- and hexapod-like silica/PS nanoparticles after up to twenty iterative silica growth steps. In all cases, one can see that the silica core grew by strictly following the shape of the PS nodules and no silica bump formed on the surface of these, confirming the relevance of the chosen multi-step synthetic approach. The diameter of the silica core increased homogeneously as a function of the successive additions of TEOS, as shown in Figure 1d,e,j,n. Morphological yields were determined by statistical analysis of lowmagnification images (Figure 1b,h,p,q) over about 100 nanoparticles. For generations \#15, yields were equal to $81 \%, 78 \%$, and $68 \%$ for bi-, tri- and hexapods, respectively. Considering the uncertainties linked to the statistical analyses, these values turn out to be very close to 
the initial morphological purity of the batches of multipods (see Table 1), which demonstrates that the regrowth stage of the silica core does not contribute to the morphological polydispersity of the batches.
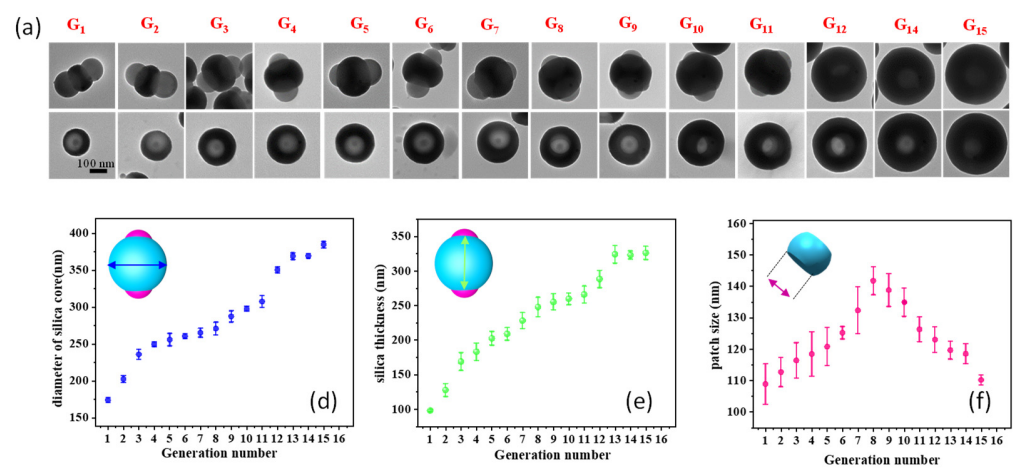

(g)
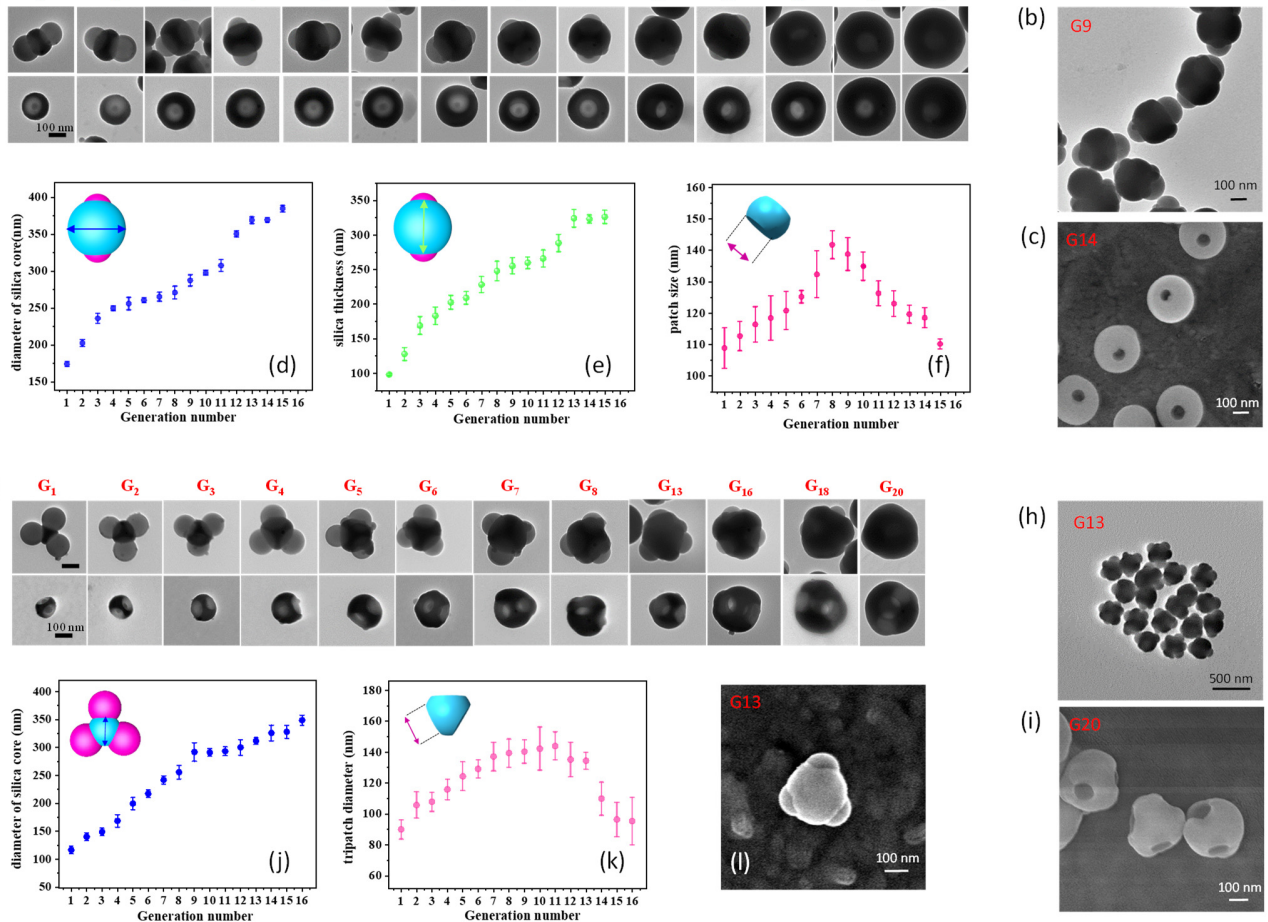

(c)

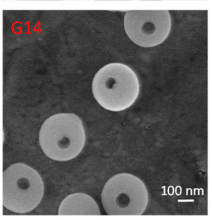

(h)

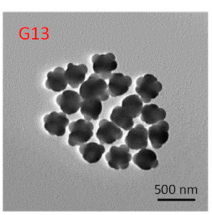

(i)
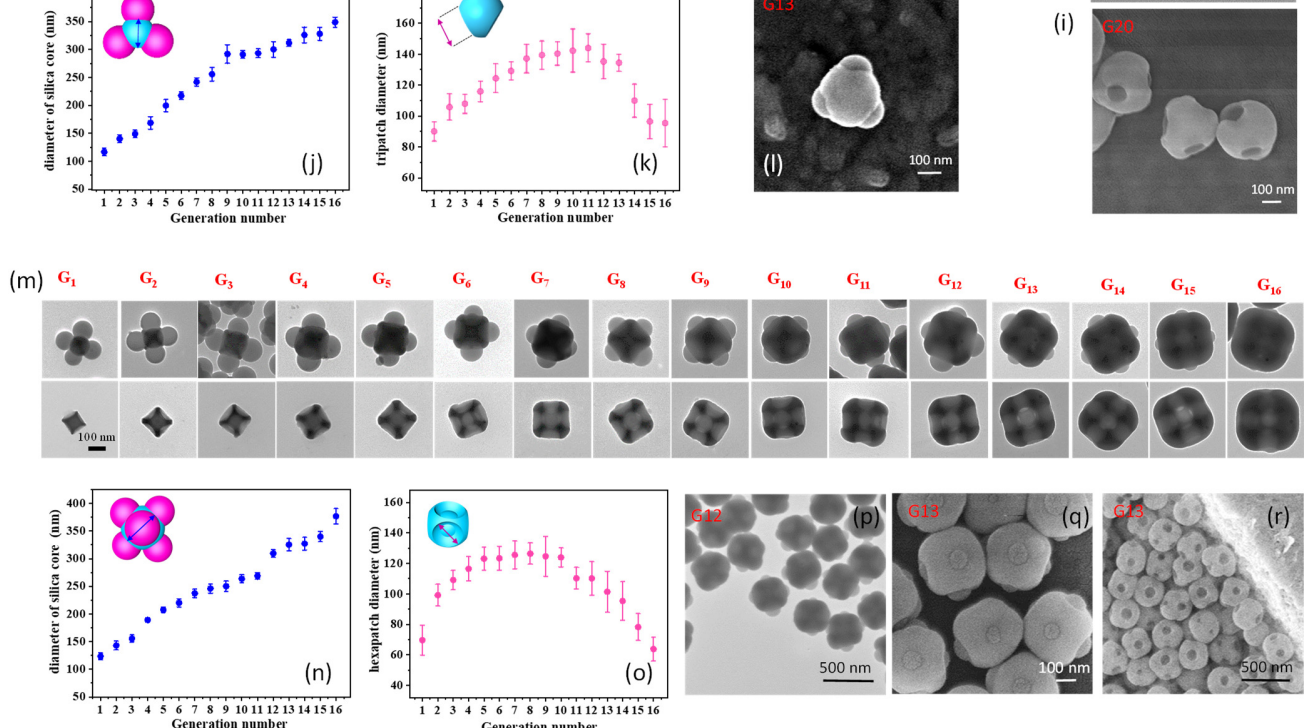

Figure 1. (a) TEM images of the bipod-like particles obtained after 1 to 15 iterative silica growth steps (corresponding to generations \#1 to \#15). The bottom row shows images of the corresponding two-patch particles obtained after dissolution of the PS nodules. All images are at the same scale; (b) Low magnification TEM image of the bipod-like particles after 9 iterative silica growth steps; (c) Scanning electron microscopy (SEM) image of the two-patch particles of generation \#14; (d,e) Evolution of the diameter and the thickness of the silica core as a function of the generation number; (f) Evolution of the patch size as a function of the generation number; (g) TEM images of the tripod-like particles obtained after 1 to 20 iterative silica growth steps. The bottom row shows images of the corresponding three-patch particles obtained after dissolution of the PS nodules. All images are at the same scale; (h) Low magnification TEM image of the tripod-like particles after 13 iterative silica growth steps; (i) SEM image of the three-patch particles of generation \#20; (j) Evolution of the diameter of the silica core as a function of the generation number; $(\mathbf{k})$ Evolution of the patch size as a function of the generation number; (l) SEM image of a tripod-like particle of generation \# 13; (m) TEM images of the hexapod-like particles obtained after 1 to 16 iterative silica growth steps. The bottom row shows images of the corresponding six-patch particles obtained after dissolution of the PS nodules. All images are at the same scale; (n) Evolution of the diameter of the silica core as a function of the generation number; (o) Evolution of the patch size as a function of the generation number; ( $p$ ) Low magnification TEM image of the hexapod-like particles after 12 iterative silica growth steps; (q) SEM image of the hexapod-like particles of generation \#13; (r) SEM image of the six-patch particles of generation \#13. 
The subsequent dissolution of the PS nodules of the multipod-like nanoparticles gave rise to silica particles with a fixed number of concave patches in a well-controlled geometry, i.e., linear, triangular or cubic (Scheme 1 and Figure $1 \mathrm{a}, \mathrm{c}, \mathrm{g}, \mathrm{i}, \mathrm{m}, \mathrm{r}$ ). The values of the concave patch size were calculated for each generation from the TEM images. Figure 1f,k,o show that the patch size first started to increase to reach a maximum and then decreased while the number of TEOS additions increased. This result confirms the conformational growth of the silica core, i.e., that the silica core encapsulated the PS nodules while it grew, as its diameter must increase until it reaches the equator of the PS lobes and decreases when this point is overpassed.

A careful examination of the TEM images of the silica nanoparticles after dissolution of the PS nodules ((Figure 1a,g,m, bottom rows) reveals the presence of a dark spot in the middle of the dimples. Thanks to energy-dispersive X-ray spectroscopy (EDX) analyses reported in a previous report, we have shown that it corresponds to the presence a few PS chains with number-average mass higher than $500,000 \mathrm{~g} \mathrm{~mol}^{-1}$, which are covalently grafted onto the initial silica seed surface [23]. We took benefit of the presence of these PS "residues" that are expected to become sticky in a good/bad solvent mixture to induce the self-assembly of the patchy silica nanoparticles. Salty water was firstly added into a twopatch silica nanoparticles (Generation \#1) dispersion in THF, in order to reduce both the electrostatic repulsions between nanoparticles due to negatively charged silanolate groups at their surface (the $\zeta$ potential of the patchy silica nanoparticles was $-42 \mathrm{mV}$ at $\mathrm{pH} 5.7$ ) and solvent quality for the PS chains. The later ones therefore formed physical bonds to minimize the free surface energy of the system $[24,25]$ and chains resulting from the side-by-side assembly of the two-patch nanoparticles were obtained after $19 \mathrm{~h}$ of incubation (Figure 2a). Some chains grew up to a few microns and contained up to $\sim 50$ building units. Branching of the chains could also be observed (red arrow) and was attributed to the presence of a small fraction ( 15\%) of three-patch nanoparticles as byproducts in the batch of two-patch nanoparticles, which act as branching points. Following the same recipe, we successfully obtained chains by incubating two-patch nanoparticles of generation \#2, but it was not possible to assemble silica nanoparticles of higher generation numbers. We assume that it is because the PS chains located at the bottom of the dimples are not long enough to interact when the silica core became too thick. This result tends to indicate that enthalpic interactions between polymer patches have a predominant role in the nanoparticle assembly and that shape-induced entropic interactions [26,27] are not sufficient to induce assembly under our experimental conditions. Short chains were also obtained by adding water into a three-patch silica nanoparticles (Generation \#1) dispersion in THF and incubating for $16 \mathrm{~h}$ (Figure 2b, blue arrows). These 1D assemblies resulted in the formation of edge-to-edge connections between the patchy silica nanoparticles. More interestingly, hexagonal entities consisting of densely packed patchy nanoparticles forming edge-to-edge and point-to-point connections were also observed (Figure 2b, green arrows). At longer incubation times, these hexagons assembled into larger two-dimensional suprastructures (Figure $2 b$, right) similar to those previously observed during the self-assembly of gold nanotriangles $[28,29]$ or beveled gold triangular nanoprisms [30], which can be considered as the early stages of the formation of a planar honeycomb lattice. Unfortunately, the presence of impurities (twoand four-patch nanoparticles) strongly hinders the formation of larger ordered patterns. As shown in Figure 2c, six-patch silica nanoparticles (Generation \#1) assembled into onedimensional assemblies which form right angles after $14 \mathrm{~h}$ of incubation into a 9/1 (v/v) $\mathrm{THF} /$ water mixture. The spacing between some patchy nanoparticles, probably caused by drying during the preparation of the TEM grid, allowed us to clearly visualize the interparticular attachment of the PS chains, which were made sticky by reducing the solvent quality. At longer times, compact arrays exhibiting a simple cubic structure could be observed (Figure 2c, right) as expected [31,32], even if the presence of impurities such as nanoparticles with four or five patches (see Table 1) strongly inhibits the extension of the ordered lattice. For both three- and six-patch nanoparticles, no assembly was observed by incubation of nanoparticles issued of generation \#3 and beyond, which confirms the 
primordial role of the enthalpic patches located at the bottom of the cavities of the silica nanoparticles. When the concentration of patchy particles was divided by two (all the more so by ten), only a few small chains (for 2- or 6-patch NPs), and hexagonal entities (for 3-patch NPs) were observed, as the probability for the NPs to collide with each other is much lower. When the concentration of NPs was multiplied by 4 , similar assemblies were observed in all cases.

(a)

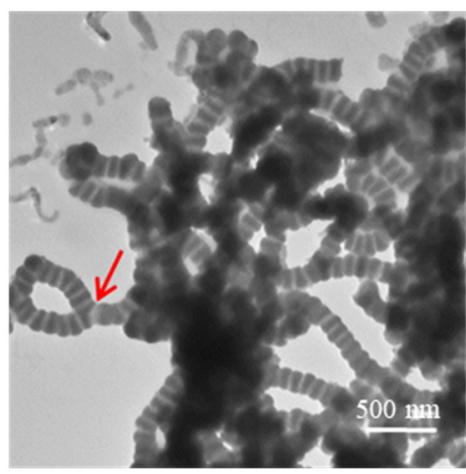

(b)

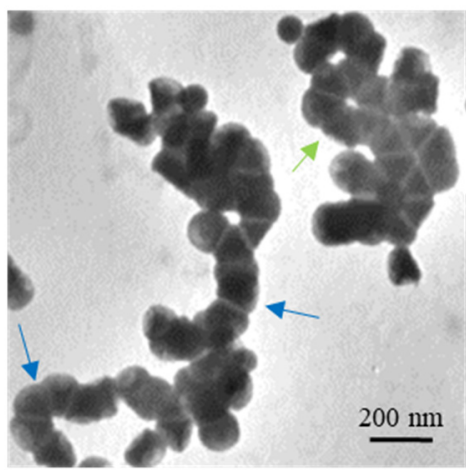

(c)

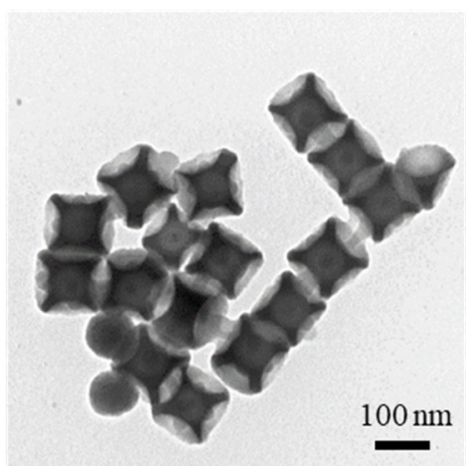

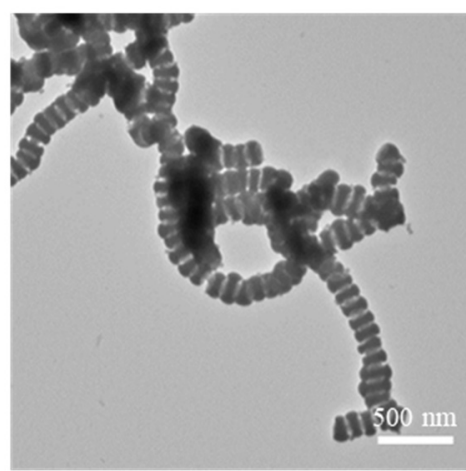
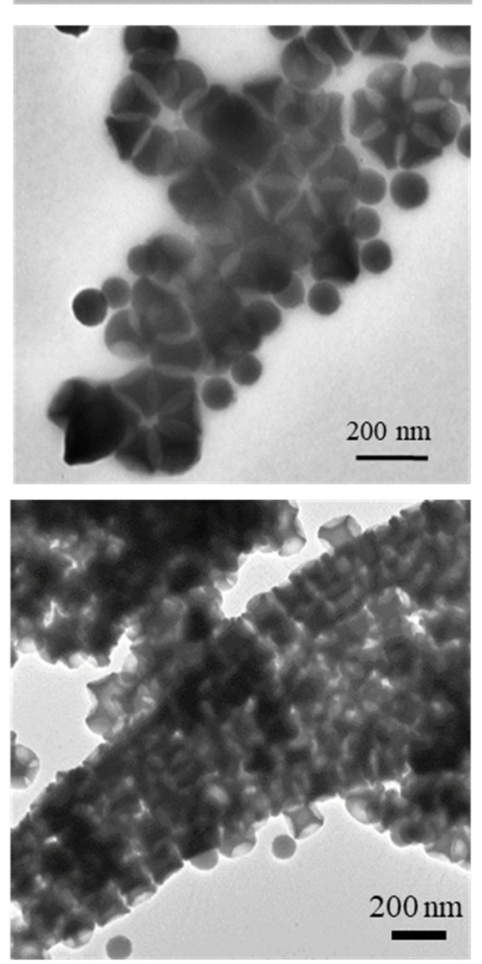

Figure 2. (a) TEM images of colloidal chains obtained after incubating two-patch silica nanoparticles for $19 \mathrm{~h}$ into a $7 / 3(v / v)$ THF/salty water mixture. (b) TEM images of chains and hexagonal superstructures obtained after incubating three-patch silica nanoparticles for 16 (left) and 40 (right) hours into a 9/1 (v/v) THF/water mixture. (c) TEM images of assemblies resulting from the incubation of six-patch nanoparticles for 16 (left) and 40 (right) hours into a 9/1 (v/v) THF/water mixture.

\section{Materials and Methods}

\subsection{Materials}

We used styrene (99\%), sodium persulfate $(99 \%)$, Symperonic ${ }^{\circledR}$ NP30, sodium dodecylsulfate (SDS, $>90 \%$ ), tetraethoxysilane (TEOS, $99 \%$ ), L-arginine $(98.5 \%$ ) and ammonium hydroxide (28-30\% in water), as we received them from Sigma Aldrich (Saint-QuentinFallavier, France). We purchased absolute ethanol from VWR International S.A.S (Fontenaysous-Bois, France) and methacryloxymethyltriethoxysilane (MMS, 98\%) from abcr GmbH (Karlsruhe, Germany) and used them as received. We systematically used ultrapure water 
with a resistivity of $18.2 \mathrm{M} \Omega \cdot \mathrm{cm}$ at $25{ }^{\circ} \mathrm{C}$ obtained from a Milli-Q system (Merck Millipore, Molsheim, France).

\subsection{Synthesis of the Silica/PS Multipods}

We prepared batches of bipods, tripods and hexapods consisting of a central silica core surrounded by two, three and six PS satellite nodules, respectively, by seededgrowth emulsion polymerization of styrene (Table 1), according to a procedure we published previously [33]. We used silica seeds previously surface-modified with MMS (0.5 molecule $\left.\cdot \mathrm{nm}^{-2}\right)$, a $95 \% / 5 \%$ w/w surfactant mixture $\left(3 \mathrm{~g} \cdot \mathrm{L}^{-1}\right)$ of Symperonic ${ }^{\circledR} \mathrm{NP30}$ and SDS, and sodium persulfate as initiator ( $0.5 \% w / w$ relative to monomer). The polymerization was performed at $70^{\circ} \mathrm{C}$ for $6 \mathrm{~h}$.

Table 1. Characteristics of the multipod-like precursors.

\begin{tabular}{|c|c|c|c|}
\hline Precursors & $\begin{array}{c}\text { Diameter of } \\
\text { Silica Core }(\mathrm{nm})\end{array}$ & $\begin{array}{c}\text { Diameter of } \\
\text { PS Nodules } \\
\text { (nm) }\end{array}$ & Morphology Purity $^{1}$ \\
\hline Bipods & $55 \pm 2$ & $160 \pm 6$ & $79 \%$ bipods, $15 \%$ tripods, $6 \%$ monopods \\
\hline Tripods & $55 \pm 2$ & $145 \pm 6$ & $77 \%$ tripods, $9 \%$ bipods, $14 \%$ tetrapods \\
\hline Hexapods & $85 \pm 2$ & $130 \pm 5$ & $81 \%$ hexapods, $15 \%$ pentapods, $4 \%$ tetrapods \\
\hline
\end{tabular}

1 determined by statistical analysis of TEM images.

\subsection{Controlled Growth of the Silica Core}

Totals of $9.1 \mathrm{~mL}$ of absolute ethanol, $0.7 \mathrm{~mL}$ of ammonia and $0.2 \mathrm{~mL}$ of the dispersion of silica-polystyrene multipods were introduced into a $25 \mathrm{~mL}$ flask and the mixture was homogenized using a magnetic bar. Then, $200 \mu \mathrm{L}$ of TEOS was added all at once after $5 \mathrm{~min}$. The reaction was left under stirring at $20^{\circ} \mathrm{C}$ for $15 \mathrm{~min}$. The reaction medium was poured into a $50 \mathrm{~mL}$ Falcon tube containing $15 \mathrm{~mL}$ of absolute ethanol. After 2 cycles of centrifugation $(12,000 \times \mathrm{g} / 5 \mathrm{~min})$ and redispersion in absolute ethanol, the nanoparticles were finally redispersed in $10 \mathrm{~mL}$ of a previously prepared hydro-alcoholic solution (absolute ethanol/ammonia/water volume ratio: $91 \% / 7 \% / 2 \%$ ). This protocol was renewed to obtain the next generation.

\subsection{Dissolution of the PS Nodules}

For dissolving the PS nodules of the multipods, $100 \mu \mathrm{L}$ of the particle dispersion was subjected to two centrifugation/redispersion cycles in THF $(8000 \times g / 10 \mathrm{~min} ; 200 \mu \mathrm{L})$. The final concentration of patchy NPs in THF was adjusted to $3.6 \cdot \times 10^{14}$ part/L and the solution was kept at $4{ }^{\circ} \mathrm{C}$.

\subsection{Assembly of the Patchy Particles}

Incubation of the particles was carried out in $15 \mathrm{~mL}$ tubes under rolling motion at $60 \mathrm{rpm}$ at room temperature. For reducing the quality of the THF solvent, a calculated volume of water for three- and six-patch nanoparticles or salty water $(20 \mathrm{mM} \mathrm{NaCl}$ aqueous solution) for two-patch nanoparticles was added drop-by-drop under stirring to reach a fraction of 10 vol.\% (for three-patch and six-patch nanoparticles) or 30 vol.\% (for two-patch nanoparticles) and a total volume of $2 \mathrm{~mL}$. It took about $20 \mathrm{~s}$ to add $100 \mu \mathrm{L}$. Assembled structures were monitored by collecting at various incubation times $50 \mu \mathrm{L}$ samples and direct deposition on TEM grids for liquid evaporation before observation.

\subsection{Characterization Techniques}

Transmission electron microscopy (TEM) and scanning electron microscopy (SEM) experiments were performed using a Hitachi $\mathrm{H} 600$ microscope operating at an acceleration voltage of $75 \mathrm{kV}$ and a Hitachi S4500 microscope with an accelerating voltage of $5 \mathrm{kV}$, respectively. We prepared the samples by depositing one drop of the colloidal dispersion on conventional carbon-coated copper grids. We let the liquid evaporate in the open air 
at room temperature and placed the grids in a box away from dust. Before SEM analysis, samples deposited on a 300-mesh carbon coated copper grid were coated with nm-thick layer of gold and palladium (10\%) with a SC7620 mini sputter coater.

The $\zeta$ potential value of a two-patch silica nanoparticles aqueous dispersion $\left(10^{15}\right.$ nanoparticles $\cdot \mathrm{L}^{-1}, \mathrm{pH}$ 5.7) was measured using the Malvern Zetasizer 3000 HS setup (Malvern Instruments, Malvern, UK). The dielectric constant of water was set to 80.4 and the Smoluchowsky constant $\mathrm{f}(\mathrm{ka})$ was 1.5 .

\section{Conclusions}

In conclusion, the conformational enlargement of the silica core of bi-, tri- and hexapods allowed us to synthesize silica nanoparticles with two, three or six circular PS patches of controlled size, respectively. The incubation of the patchy nanoparticles in a mixture of a good and a bad solvent for PS made the patches sticky, which induced the assembly of the patchy silica nanoparticles in the form of chains, hexagonal suprastructures and simple cubic lattice depending on the number of patches at their surface. Even if homogeneity and purity of the patchy nanoparticles are major challenges, which must be taken into account, extending such a strategy to mixtures of particles with different numbers of patches could lead to the fabrication of new nonconventional ordered assemblies such as quasicrystals and thus contribute to enriching diversity in self-assembly structures. Another prospect concerns the direct imaging of patchy nanoparticle self-assembly in liquid, which could help to better understand the interparticle interaction governing dynamic assembly process. Liquid-phase TEM technique can be a powerful tool to achieve this challenging perspective.

Author Contributions: Conceptualization, E.D. and S.R.; investigation, B.L. and S.E.; formal analysis, B.L., E.D. and S.R.; writing-original draft preparation, B.L.; writing-review and editing, E.D. and S.R.; supervision, E.D. and S.R.; funding acquisition, E.D. and S.R. All authors have read and agreed to the published version of the manuscript.

Funding: This research received no external funding.

Institutional Review Board Statement: Not applicable.

Informed Consent Statement: Not applicable.

Data Availability Statement: The data presented in this study are available on request from the corresponding authors.

Acknowledgments: B.L. gratefully acknowledges the China Scholarship Council for his PhD grant.

Conflicts of Interest: The authors declare no conflict of interest.

Sample Availability: Samples of the patchy silica nanoparticles are available from the authors.

\section{References}

1. Adnan, M.M.; Dalod, A.R.M.; Balci, M.H.; Glaum, J.; Einarsrud, M.A. In situ synthesis of hybrid inorganic-polymer nanocomposites. Polymers 2018, 10, 1129. [CrossRef]

2. Faustini, M. Sol-gel engineering to tune structural colours. J. Sol-Gel Sci. Technol. 2020, 95, 504-516. [CrossRef]

3. Erigoni, A.; Diaz, U. Porous silica-based organic-inorganic hybrid catalysts: A review. Catalysts 2021, 11, 79. [CrossRef]

4. Thorkelsson, K.; Bai, P.; Xu, T. Self-assembly and applications of anisotropic nanomaterials: A review. Nano Today 2015, 10, 48-66. [CrossRef]

5. Morphew, D.; Chakrabarti, D. Clusters of anisotropic colloidal particles: From colloidal molecules to supracolloidal structures. Curr. Opin. Colloid Interface Sci. 2017, 30, 70-80. [CrossRef]

6. Liu, B.; Wu, Y.; Zhao, S. Anisotropic colloids: From non-templated to patchy templated synthesis. Chem. Eur. J. 2018, 24, 10562-10570. [CrossRef]

7. Xu, W.; Li, Z.; Yin, Y. Colloidal assembly approaches to micro/nanostructures of complex morphologies. Small 2018, 14, 1801083. [CrossRef]

8. Su, Z.; Zhang, R.; Yan, X.Y.; Guo, Q.Y.; Huang, J.; Shan, W.; Liu, Y.; Huang, M.; Cheng, S.Z.D. The role of architectural engineering in macromolecular self-assemblies via non-covalent interactions: A molecular LEGO approach. Prog. Polym. Sci. 2020, 103, 101230. [CrossRef] 
9. Bouju, X.; Duguet, E.; Gauffre, F.; Henry, C.R.; Kahn, M.L.; Mélinon, P.; Ravaine, S. Non-isotropic self-assembly of nanoparticles: From compact packing to functional aggregates. Adv. Mater. 2018, 30, 1706558. [CrossRef]

10. Tréguer-Delapierre, M.; Madeira, A.; Hubert, C.; Ravaine, S. Recent advances in the synthesis of anisotropic particles. In Anisotropic Particle Assemblies; Wu, N., Lee, D., Striolo, A., Eds.; Elsevier: Amsterdam, The Netherlands, 2018; pp. 1-35. [CrossRef]

11. Anu Mary Ealia, S.; Saravanakumar, M.P. A review on the classification, characterisation, synthesis of nanoparticles and their application. IOP Conf. Ser. Mater. Sci. Eng. 2017, 263, 032019. [CrossRef]

12. He, M.; Gales, J.P.; Ducrot, E.; Gong, Z.; Yi, G.R.; Sacanna, S.; Pine, D.J. Colloidal diamond. Nature 2020, 585, 524-529. [CrossRef]

13. Pazos-Perez, N.; Wagner, C.S.; Romo-Herrera, J.M.; Liz-Marzán, L.M.; García de Abajo, F.J.; Wittemann, A.; Fery, A.; AlvarezPuebla, R.A. Organized plasmonic clusters with high coordination number and extraordinary enhancement in surface-enhanced Raman scattering (SERS). Angew. Chem. Int. Ed. 2012, 51, 12688-12693. [CrossRef]

14. Schrade, A.; Cao, Z.; Landfester, K.; Ziener, U. Preparation of raspberry-like nanocapsules by the combination of Pickering emulsification and solvent displacement technique. Langmuir 2011, 27, 6689-6700. [CrossRef] [PubMed]

15. Zoldesi, C.I.; Imhof, A. Synthesis of monodisperse colloidal spheres, capsules, and microballoons by emulsion templating. Adv. Mater. 2005, 17, 924-928. [CrossRef]

16. Wang, B.; Chen, J.S.; Wu, H.B.; Wang, Z.; Lou, X.W. Quasiemulsion-templated formation of $\alpha$-Fe $\mathrm{F}_{2} \mathrm{O}_{3}$ hollow spheres with enhanced lithium storage properties. J. Am. Chem. Soc. 2011, 133, 17146-17148. [CrossRef] [PubMed]

17. Lu, Y.; McLellan, J.; Xia, Y. Synthesis and crystallization of hybrid spherical colloids composed of polystyrene cores and silica shells. Langmuir 2004, 20, 3464-3470. [CrossRef]

18. Joo, B.; Zhang, Q.; Lee, I.; Dahl, M.; Zaera, F.; Yin, Y. Mesoporous anatase titania hollow nanostructures though silica-protected calcination. Adv. Funct. Mater. 2012, 22, 166-174. [CrossRef]

19. Chen, T.; Chen, G.; Xing, S.; Wu, T.; Chen, H. Scalable routes to Janus $\mathrm{Au}-\mathrm{SiO}_{2}$ and ternary $\mathrm{Ag}-\mathrm{Au}-\mathrm{SiO} 2$ nanoparticles. Chem. Mater. 2010, 22, 3826-3828. [CrossRef]

20. Li, W.; Palis, H.; Mérindol, R.; Majimel, J.; Ravaine, S.; Duguet, E. Colloidal molecules and patchy particles: Complementary concepts, synthesis and self-assembly. Chem. Soc. Rev. 2020, 49, 1955-1976. [CrossRef] [PubMed]

21. Désert, A.; Hubert, C.; Fu, Z.; Moulet, L.; Majimel, J.; Barboteau, P.; Thill, A.; Lansalot, M.; Bourgeat-Lami, E.; Duguet, E.; et al. Synthesis and site-specific functionalization of tetravalent, hexavalent, and dodecavalent silica particles. Angew. Chem. Int. Ed. 2013, 52, 11068-11072. [CrossRef]

22. Rouet, P.E.; Khalaf, R.; Exiga, S.; Duguet, E.; Ravaine, S. Synthesis of tetrahedral patchy nanoparticles with controlled patch size. J. Nanopart. Res. 2020, 22, 337. [CrossRef]

23. Hubert, C.; Chomette, C.; Désert, A.; Sun, M.; Tréguer-Delapierre, M.; Mornet, S.; Perro, A.; Duguet, E.; Ravaine, S. Synthesis of multivalent silica nanoparticles combining both enthalpic and entropic patchiness. Faraday Discuss. 2015, 181, 139-146. [CrossRef]

24. Liu, K.; Nie, Z.; Zhao, N.; Li, W.; Rubinstein, M.; Kumacheva, E. Step-growth polymerization of inorganic nanoparticles. Science 2010, 329, 197-200. [CrossRef]

25. Choueiri, R.M.; Galati, E.; Klinkova, A.; Thérien-Aubin, H.; Kumacheva, E. Linear assembly of patchy and non-patchy nanoparticles. Faraday Discuss. 2016, 191, 198-204. [CrossRef]

26. Van Anders, G.; Ahmed, N.K.; Smith, R.; Engel, M.; Glotzer, S.C. Entropically patchy particles: Engineering valence through shape entropy. ACS Nano 2014, 8, 931-940. [CrossRef]

27. Millan, J.A.; Ortiz, D.; Glotzer, S.C. Effect of shape on the self-assembly of faceted patchy nanoplates with irregular shape into tiling patterns. Soft Matter. 2015, 11, 1386-1396. [CrossRef]

28. Fu, Q.; Ran, G.; Xu, W. Direct self-assembly of CTAB-capped Au nanotriangles. Nano Res. 2016, 9, 3247-3256. [CrossRef]

29. Walker, D.A.; Browne, K.P.; Kowalczyk, B.; Grzybowski, B.A. Self-assembly of nanotriangle superlattices facilitated by repulsive electrostatic interactions. Angew. Chem. Int. Ed. 2010, 49, 6760-6763. [CrossRef] [PubMed]

30. Kim, J.; Song, X.; Ji, F.; Luo, B.; Ice, N.F.; Liu, Q.; Zhang, Q.; Chen, Q. Polymorphic assembly from beveled gold triangular nanoprisms. Nano Lett. 2017, 17, 3270-3275. [CrossRef] [PubMed]

31. Agarwal, U.; Escobedo, F. Mesophase behaviour of polyhedral particles. Nat. Mater. 2011, 10, 230-235. [CrossRef] [PubMed]

32. Du, C.X.; van Anders, G.; Newman, R.S.; Glotzer, S.C. Shape-driven solid-solid transitions in colloids. Proc. Natl. Acad. Sci. USA 2017, 114, E3892-E3899. [CrossRef] [PubMed]

33. Désert, A.; Morele, J.; Taveau, J.C.; Lambert, O.; Lansalot, M.; Bourgeat-Lami, E.; Thill, A.; Spalla, O.; Belloni, L.; Ravaine, S.; et al. Multipod-like silica/polystyrene clusters. Nanoscale 2016, 8, 5454-5469. [CrossRef] [PubMed] 